\title{
Hybrid silicon on silicon carbide integrated photonics platform
}

Cite as: Appl. Phys. Lett. 115, 141105 (2019); https://doi.org/10.1063/1.5116201

Submitted: 22 June 2019 . Accepted: 19 August 2019 . Published Online: 02 October 2019

Chuting Wang (D), Evan Miyazono, Ioana Craiciu, and Andrei Faraon

\section{COLLECTIONS}

F This paper was selected as Featured
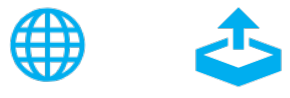

\section{Lock-in Amplifiers up to $600 \mathrm{MHz}$}

starting at

$\$ 6,210$

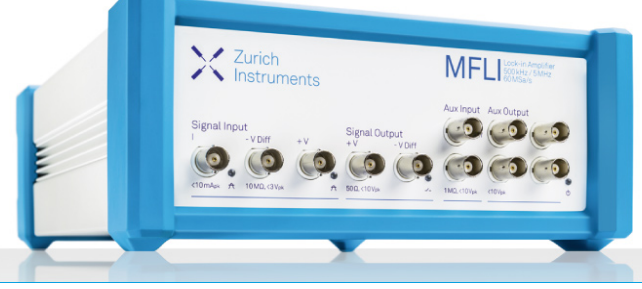

Zurich

- Instruments

Watch the Video 


\title{
Hybrid silicon on silicon carbide integrated photonics platform 8 (c)
}

\author{
Cite as: Appl. Phys. Lett. 115, 141105 (2019); doi: 10.1063/1.5116201 \\ Submitted: 22 June 2019 • Accepted: 19 August 2019 . \\ Published Online: 2 October 2019
}

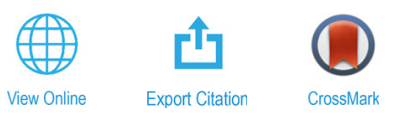

\author{
Chuting Wang, (D) Evan Miyazono, loana Craiciu, and Andrei Faraon ${ }^{\text {a) }}$
}

\begin{abstract}
AFFILIATIONS
Department of Applied Physics and Kavli Nanoscience Institute, California Institute of Technology, 1200 E California Blvd., Pasadena, California 91125, USA
\end{abstract}

a)faraon@caltech.edu

\begin{abstract}
We demonstrate a hybrid on-chip photonics platform based on crystalline silicon resonators and waveguides patterned on top of silicon carbide. The devices were fabricated with membrane transfer followed by standard electron beam patterning procedures. The platform allows the integration of high quality silicon photonics with color centers in silicon carbide operating in the near infrared for spin-photon interfaces used in quantum information processing applications. We measure waveguide-coupled ring resonators with loaded quality factors up to 23000 at cryogenic temperatures.
\end{abstract}

Published under license by AIP Publishing. https://doi.org/10.1063/1.5116201

A variety of solid-state single photon emitters act as promising optically addressable spin qubits for photonic quantum networks. ${ }^{1-3}$ In these networks, it is important that emitters produce indistinguishable photons. This process can be improved by using scalable on-chip cavity devices that couple to the optical transition to generate lifetime limited emission and channel it into optical waveguides. ${ }^{4-6}$ One strategy to develop on-chip photonic devices is to fabricate them directly in the material of interest. For this technique, it is important to start with thin membranes on a low refractive index substrate or on a substrate that can be etched away or undercut. Even if some techniques exist to produce membranes, it is not always the case that these membranes can host high quality quantum emitters. An alternative is to make hybrid devices in a high refractive index layer located on top of the substrate hosting the qubits, such that the emitters are coupled to the evanescent field.

In this paper, we demonstrate a crystalline silicon (c-Si) hybrid platform of ring resonators and waveguides fabricated on top of the $\mathrm{SiC}$ substrate. Based on a Si membrane transfer technique, these hybrid resonators can be fabricated on different substrates for coupling to a variety of emitters operating in the transparency region of silicon in the infrared. In this work, we fabricated resonators for coupling to a (kh) subset of $4 \mathrm{H}-\mathrm{SiC}$ divacancies close to the surface for scalable spinphoton interface applications [Figs. 1(a) and 1(b)]. These devices will combine the excellent quality of silicon photonics and the very promising quantum defects in SiC. Color centers like neutrally charged divacancies in silicon carbide $(\mathrm{SiC})$ have attracted attention in recent years. Their spin states can be initialized and manipulated via microwaves ${ }^{7,8}$ or optics, ${ }^{9}$ and in terms of optical and spin properties, they possess similar properties to negatively charged nitrogen vacancy (NV) centers in diamond. ${ }^{10-12}$ The (kh) divacancy has the optical zero phonon line (ZPL) in near infrared close to $1080 \mathrm{~nm}$, but the ZPL emission is only around $10 \%$ of the total emission ${ }^{13}$ and enhancement of ZPL is crucial to achieve a higher generation rate of indistinguishable photons. A similar hybrid approach with gallium phosphide as the high index material on top of diamond was employed for coupling to the ZPL emission of NV centers, ${ }^{14,15}$ and hybrid crystalline resonators on yttrium orthosilicate have been coupled to erbium atoms. ${ }^{16}$

The devices investigated in this work are composed of 3 grating couplers and 2 ridge waveguides coupled to a ring resonator [Fig. 1 (a) ]. ${ }^{17}$ This design allows for measurements using the drop port and is convenient for characterizing the resonance by measuring either the light transmitted from one waveguide to the other through the ring or the transmission through one ring-coupled waveguide. One of the waveguide ends is bent at $90^{\circ}$ to allow for cross-polarized measurements with higher extinction between input and output light when using a confocal microscope to image the sample. One of the waveguides has a gradually tapered end instead of a grating coupler to reduce reflection that causes interference on the measured signal. The small thin feature near the bent waveguide is used to block any spurious light escaping the input waveguide from hitting the grating coupler. The design of the rings is verified with finite-difference time-domain simulations using the open source software MEEP. ${ }^{18}$ The parameters for the ring width and height are chosen to optimize the fundamental transverse magnetic (TM) mode, first 
(a)

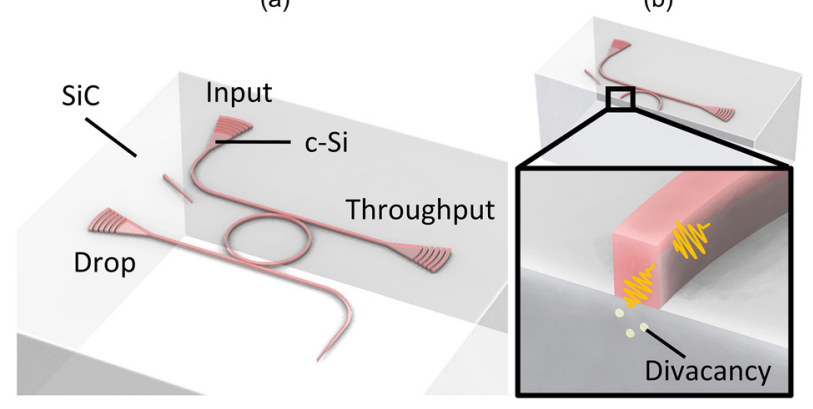

FIG. 1. (a) A schematic of the c-Si ring resonator on $4 \mathrm{H}-\mathrm{SiC}$ for spin-photon interfaces. C-Si is drawn in red, while the transparent part underneath is $4 \mathrm{H}-\mathrm{SiC}$. (b) Cross section showing the ring resonator near color centers in the $4 \mathrm{H}-\mathrm{SiC}$ underneath it, which can couple to the evanescent field of the cavity.

with 2D axisymmetric simulations [Figs. 2(a)-2(d)] followed by 3D simulations [Figs. 2(e)-2(g)]. The TM mode was chosen because a stronger field can exist beneath the $\mathrm{Si}$ and $\mathrm{SiC}$ interface compared to the transverse electric mode due to the field discontinuity [Fig. 2(c)]. The field amplitude at the defect linearly scales with the spontaneous emission enhancement factor. ${ }^{14,19}$ First, we simulate the intrinsic quality factor $(\mathrm{Q})$ of the rings without the waveguides and choose a ring with the smallest radius, which still supported modes with high quality factors [Fig. 2(d)]. The parameters we choose for the ring are a radius of $3.75 \mu \mathrm{m}$, a height of $360 \mathrm{~nm}$, and a width of $300 \mathrm{~nm}$, which give a simulated intrinsic quality factor of $\mathrm{Q}=7 \times 10^{5}$. The radius was chosen to minimize the ratio of the quality factor to mode volume considering that the quality factor in the current fabricated devices is limited by scattering losses. Next, we introduce symmetric waveguides with a width of $300 \mathrm{~nm}$ to the simulation [Figs. 2(e) and 2(f)] and simulate the loaded quality factor by varying the separation between the waveguides and the ring [Fig. 2(g)]. Based on this simulation, we fabricate arrays of rings that range from overcoupled to undercoupled by (a)

(b)

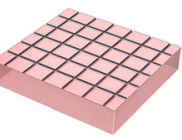

(d)

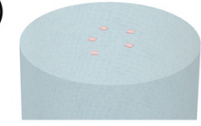

$\uparrow$

(e)

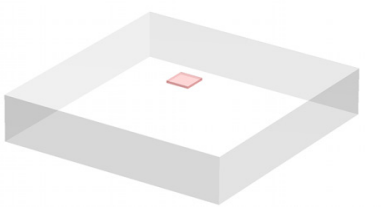

(f)

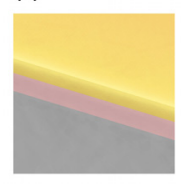

(g)

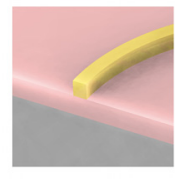

(h)

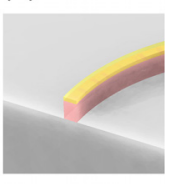

(i)

FIG. 3. (a)-(e) A schematic of the membrane transfer procedure: (a) $500 \mu \mathrm{m}$ $\times 500 \mu \mathrm{m}$ c-Si membranes are patterned on an SOI chip. (b) HF wet etching detaches the membranes from the handle Si layer. (c) Transferring the wet etched sample into water exfoliates the membranes. (d) Picking up a single membrane with the SiC substrate. (e) After drying, the membrane attaches firmly to the substrate. (f)-(i) A schematic of the ring resonator patterning procedure (cross-sectional view). (f) Spin coated ZEP 520 (yellow) on c-Si (red) and 4H-SiC (gray). (g) E-beam lithography writing to pattern the resist in the shape of ring resonators. (h) Using the resist as an etching mask, the sample is etched to transfer the pattern to the c-Si layer. (i) Final cleaning with remover PG followed by removal of the resist by oxygen plasma.

changing the waveguide-ring separation $(110 \mathrm{~nm}-230 \mathrm{~nm})$. We obtain resonance spreading over the free spectral range of $\sim 13 \mathrm{~nm}$ close to $1080 \mathrm{~nm}$ by slightly modifying the ring radius so that some of them could be tuned onto ZPL of the divacancy by gas deposition for coupling to the defects. ${ }^{17}$

The device fabrication procedure consists of transferring c-Si membranes followed by standard silicon patterning processes (Fig. 3). The c-Si membrane transfer involves wet etching, membrane release, and pickup. The process starts by first creating a $500 \mu \mathrm{m} \times 500 \mu \mathrm{m}$ c-Si square from a silicon-on-oxide (SOI) chip by standard photolithography and dry etching [Fig. 3(a)]. Then, wet etching in HF

(a)

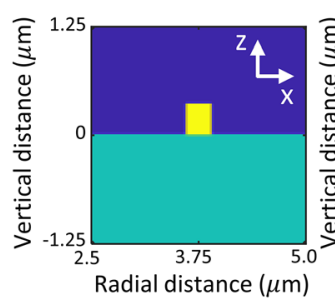

(e)

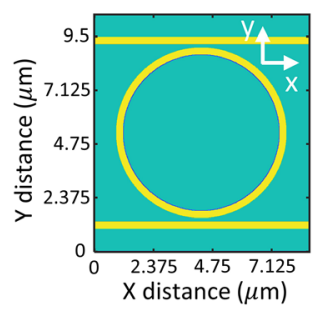

(b)

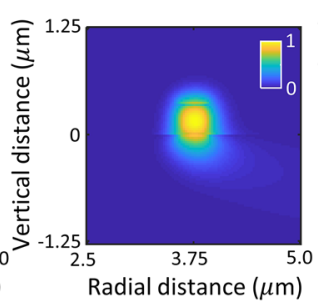

(f)

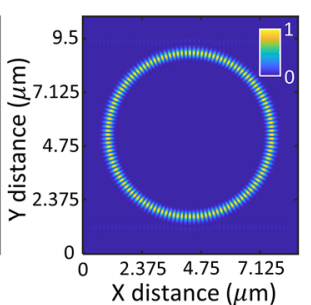

(c)

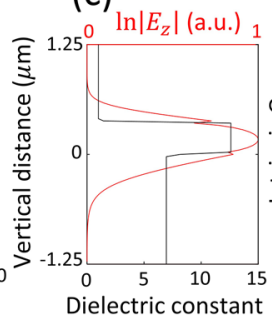

(g)

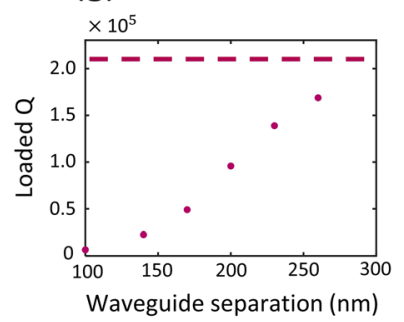

(d)

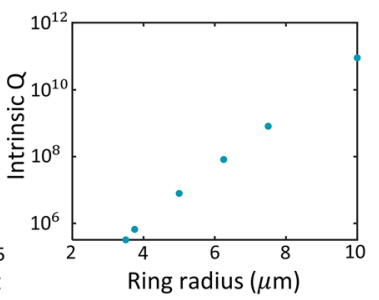

FIG. 2. Simulations of $\mathrm{c}-\mathrm{Si}$ on $\mathrm{SiC}$ ring resonators. (a) Refractive index profile in the $2 \mathrm{D}$ axisymmetric configuration. (yellow: $\mathrm{N}_{\mathrm{Si}}=3.5$, green: $\mathrm{N}_{\mathrm{SiC}}=2.6$, and blue: $\mathrm{N}_{\text {air }}=1.0$ ) (b) The TM mode (polarized in the $z$ direction) electric field amplitude profile In $\left|E_{z}\right|$ for an uncoupled ring. (c) In $\left|E_{z}\right|$ and refractive index profile along the centerline. (d) Simulated intrinsic quality factor vs ring radius for a c-Si ring with a height of $360 \mathrm{~nm}$, a width of $300 \mathrm{~nm}$, and a radius of $3.75 \mu \mathrm{m}$ in the $2 \mathrm{D}$ axisymmetric configuration. (e) Refractive index profile used in the 3D simulation. (f) $\ln \left|E_{z}\right|$ for a waveguide-coupled ring resonator. (g) Simulated loaded quality factor vs waveguide separation with the same dimensions. The dashed line indicates the intrinsic $Q$ simulated without waveguide in the $3 \mathrm{D}$ configuration. 
removes the oxide layer and c-Si membranes lie detached on the $\mathrm{Si}$ handle layer [Fig. 3(b)]. By moving the chip to water, the c-Si membranes are exfoliated and float on the water surface [Fig. 3(c)]. The $4 \mathrm{H}-\mathrm{SiC}$ samples (from CREE, Inc.) were cleaned using the mixture of sulfuric acid and hydrogen peroxide (Nano-Strip) followed by treatment with oxygen plasma. We found that oxygen plasma cleaning of the substrate made a significant difference in achieving good attachment of the membranes to the substrate. The membranes were picked up from the water with the clean $\mathrm{SiC}$ substrate [Fig. 3(d)]. After naturally drying the residual water on the $\mathrm{SiC}$ sample, the membrane bonded strongly with the substrate by Van der Waals adhesion and rinsing or spin coating did not wash the membrane away. After a single membrane was transferred [Fig. 3(e)], we were able to repeat the membrane picking up process with the same substrate to transfer multiple membranes on it. This simple method with a high yield is inspired by more complicated methods for obtaining larger membranes. ${ }^{20}$

After the c-Si membrane transfer, we write and etch the rings using a standard fabrication process. We first spin coat ZEP520 resist [Fig. 3(f)], followed by e-beam lithography patterning, where the resist is patterned with ring resonators [Fig. $3(\mathrm{~g})]$. Then, the sample is etched by pseudobosch plasma enhanced reactive ion etching to transfer the pattern to the c-Si layer [Fig. 3(h)]. Finally, the sample is cleaned with $\mathrm{N}$-methyl-2-pyrrolidone (NMP) based solvent remover PG overnight followed by oxygen plasma and solvent rinsing to remove the resist [Fig. 3(i)]. The final fabricated ring resonator device is shown in Fig. 4.

Device measurements are performed using a home-built confocal microscope setup that measures samples located in a helium flow cryostat. Operation at low temperatures is necessary to shift the silicon band edge toward shorter wavelengths and thus enable the achievement of high quality resonances at wavelengths around $1078 \mathrm{~nm}$ where one of the di-vacancies has its zero phonon line. A $50 \times$ microscope objective (Olympus LCPLN50XIR) is used for coupling light into the waveguide and for collecting the light diffracted from the grating couplers. A pinhole is used on the output as a spatial filter, blocking all light except from the region around the output grating coupler. The collected output signal through the drop port is first measured using a supercontinuum source and a near infrared grating spectrometer with a resolution of $9.0 \mathrm{GHz}$ [Fig. 5(a)]. The signal from a throughput port has an interference pattern from reflection between grating couplers, which makes it more difficult to observe when the ring

(a)

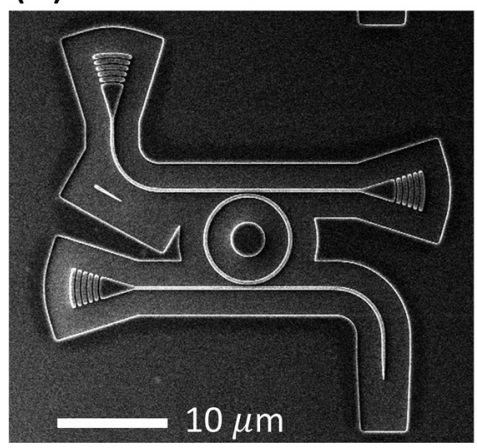

FIG. 4. Scanning electron microscopy image of (a) a ring resonator device, (b) a grating coupler, and (c) ring sidewalls around the waveguide-ring coupling region.
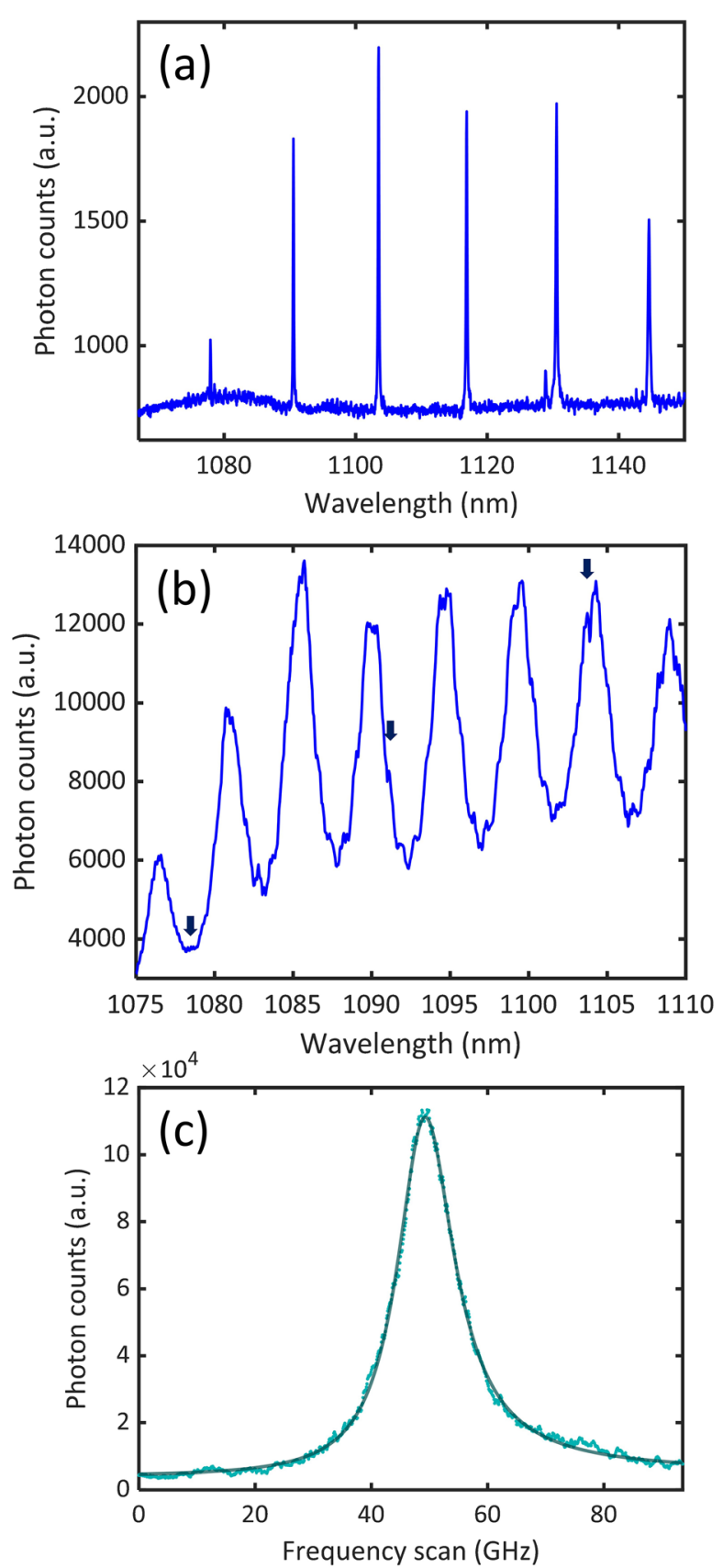

FIG. 5. (a) Transmission spectrum through a drop port. Resonances lie from 1060 to $1250 \mathrm{~nm}$ at $20 \mathrm{~K}$. (b) Transmission spectrum through a throughput port at $20 \mathrm{~K}$. The blue arrows indicate the locations of resonances. (c) Resonance with the Lorentzian fit shows a linewidth of $12 \mathrm{GHz}$, corresponding to a quality factor of about 23000 at $20 \mathrm{~K}$ and a wavelength of $1078 \mathrm{~nm}$.

resonators are undercoupled [Fig. 5(b)]. Resonances with a linewidth close to the spectrometer resolution are measured. To better quantify the quality factor of the resonances, we use an external cavity tunable laser with a resolution of $\sim 95 \mathrm{MHz}$. Using this method, the best 
quality factor we achieved was $\sim 23000$ with a Lorentzian fit at $20 \mathrm{~K}$ and at a wavelength of $1078 \mathrm{~nm}$ [Fig. 5(c)]. The dimensions of the best measured ring resonator are a radius of $3.75 \mu \mathrm{m}$, a height of $360 \mathrm{~nm}$, a width of $300 \mathrm{~nm}$, and a waveguide-ring separation of $230 \mathrm{~nm}$. The quality factor of the same device was measured to be $\sim 11000$ at room temperature, indicating that $\mathrm{Si}$ absorption becomes the dominant loss.

Based on simulation and measurements, the mode volume of the ring resonator is $19.5\left(\lambda / n_{s i}\right)^{3}$ and the best measured quality factor is 23000 at a wavelength of $1078 \mathrm{~nm}$. This would result in a Purcell enhancement factor of $\sim 36$ assuming perfect dipole alignment ${ }^{19}$ for an emitter located at $10 \mathrm{~nm}$ below the surface. The estimated Purcell enhancement factor for an emitter at a depth of $100 \mathrm{~nm}$ is $\sim 12$ due to a 3 times smaller field [Fig. 2(c)]. These parameters are sufficient for demonstrating proof-of-concept coupling of divacancies to on-chip silicon resonators.

We demonstrated a fabrication method for Si hybrid ring resonator devices, which can be used for coupling quantum emitters close to the surface of the underneath substrate. The transferrable Si membrane fabrication technique can be applied to enhance emission of different qubit candidates. These devices will find future usage in scalable on-chip quantum light matter interfaces based on infrared color centers at cryogenic temperature. Future effort would focus on increasing the quality factor of the devices by improving the fabrication procedures and developing photonic devices like photonic crystal resonators that have smaller mode volumes, leading to increased strength of the light-matter interaction.

The authors would like to thank Yu Horie, Jake Rochman, Ehsan Arbabi, and Seyedeh Mahsa Kamali for discussions on fabrication techniques as well as Jon Kindem for discussions on setting up an external cavity tunable laser. We acknowledge funding from AFOSR Quantum Transduction Multidisciplinary University Research Initiative (No. FA9550-15-1-002). C.W. acknowledges support from Japan Student Support Organization (JASSO). I.C. acknowledges support from the Natural Sciences and Engineering Research Council of Canada (NSERC) (No. PGSD2-502755-2017).

\section{REFERENCES}

${ }^{1}$ H. J. Kimble, “The quantum internet," Nature 453, 1023-1030 (2008).

${ }^{2}$ J. L. O’Brien, A. Furusawa, and J. Vučković, "Photonic quantum technologies," Nat. Photonics 3(12), 687-695 (2009).

${ }^{3}$ C. Simon, “Towards a global quantum network," Nat. Photonics 11(11), 678 (2017).

${ }^{4}$ G. Calusine, A. Politi, and D. D. Awschalom, "Cavity-enhanced measurements of defect spins in silicon carbide,” Phys. Rev. Appl. 6(1), 014019 (2016).
${ }^{5}$ L. Li, T. Schröder, E. H. Chen, M. Walsh, I. Bayn, J. Goldstein, O. Gaathon, M. E. Trusheim, M. Lu, J. Mower, M. Cotlet, M. L. Markham, D. J. Twitchen, and D. Englund, "Coherent spin control of a nanocavity-enhanced qubit in diamond," Nat. Commun. 6, 6173 (2015).

${ }^{6}$ D. O. Bracher, X. Zhang, and E. L. Hu, "Selective purcell enhancement of two closely linked zero-phonon transitions of a silicon carbide color center," Proc. Natl. Acad. U.S.A. 114(16), 4060-4065 (2017).

${ }^{7}$ W. F. Koehl, B. B. Buckley, F. J. Heremans, G. Calusine, and D. D. Awschalom, "Room temperature coherent control of defect spin qubits in silicon carbide," Nature 479(7371), 84-87 (2011).

${ }^{8}$ D. J. Christle, P. V. Klimov, C. F. de las Casas, K. Szász, V. Ivády, V. Jokubavicius, J. Ul Hassan, M. Syväjärvi, W. F. Koehl, T. Ohshima, N. T. Son, E. Janzén, Á. Gali, and D. D. Awschalom, "Isolated spin qubits in SiC with a high-fidelity infrared spin-to-photon interface," Phys. Rev. X 7(2), 021046 (2017).

${ }^{9}$ O. V. Zwier, D. O'Shea, A. R. Onur, and C. H. van der Wal, "All-optical coherent population trapping with defect spin ensembles in silicon carbide," Sci. Rep. 5, 10931 (2015).

${ }^{10}$ D. J. Christle, A. L. Falk, P. Andrich, P. V. Klimov, J. U. Hassan, N. T. Son, E. Janzén, T. Ohshima, and D. D. Awschalom, "Isolated electron spins in silicon carbide with millisecond coherence times," Nat. Mater. 14(2), 160-163 (2015).

${ }^{11}$ H. Seo, A. L. Falk, P. V. Klimov, K. C. Miao, G. Galli, and D. D. Awschalom, "Quantum decoherence dynamics of divacancy spins in silicon carbide," Nat. Commun. 7, 12935 (2016).

${ }^{12}$ C. F. de las Casas, D. J. Christle, J. Ul Hassan, T. Ohshima, N. T. Son, and D. D. Awschalom, "Stark tuning and electrical charge state control of single divacancies in silicon carbide," Appl. Phys. Lett. 111(26), 262403 (2017).

${ }^{13}$ K. C. Miao, A. Bourassa, C. P. Anderson, S. J. Whiteley, A. L. Crook, S. L. Bayliss, G. Wolfowicz, G. Thiering, P. Udvarhelyi, V. Ivady, H. Abe, T. Ohshima, A. Gali, and D. D. Awschalom, "Electrically driven optical interferometry with spins in silicon carbide," preprint arXiv:1905.12780 (2019).

${ }^{14}$ C. Santori, P. E. Barclay, K.-M. C. Fu, R. G. Beausoleil, S. Spillane, and M. Fisch, "Nanophotonics for quantum optics using nitrogen-vacancy centers in diamond," Nanotechnology 21(27), 274008 (2010).

${ }^{15}$ E. R. Schmidgall, S. Chakravarthi, M. Gould, I. R. Christen, K. Hestroffer, F. Hatami, and K.-M. C. Fu, "Frequency control of single quantum emitters in integrated photonic circuits," Nano Lett. 18(2), 1175-1179 (2018).

${ }^{16}$ A. M. Dibos, M. Raha, C. M. Phenicie, and J. D. Thompson, "Atomic source of single photons in the telecom band," Phys. Rev. Lett. 120(24), 243601 (2018).

${ }^{17}$ E. Miyazono, T. Zhong, I. Craiciu, J. M. Kindem, and A. Faraon, "Coupling of erbium dopants to yttrium orthosilicate photonic crystal cavities for on-chip optical quantum memories," Appl. Phys. Lett. 108(1), 011111 (2016).

${ }^{18}$ A. F. Oskooi, D. Roundy, M. Ibanescu, P. Bermel, J. D. Joannopoulos, and S. G. Johnson, "Meep: A flexible free-software package for electromagnetic simulations by the FDTD method," Comput. Phys. Commun. 181(3), 687-702 (2010).

${ }^{19}$ E. M. Purcell, H. C. Torrey, and R. V. Pound, "Resonance absorption by nuclear magnetic moments in a solid," Phys. Rev. 69(1-2), 37-38 (1946).

${ }^{20}$ L. Li, I. Bayn, M. Lu, C.-Y. Nam, T. Schröder, A. Stein, N. C. Harris, and D. Englund, "Nanofabrication on unconventional substrates using transferred hard masks," Sci. Rep. 5, 7802 (2015). 